\title{
GUT inspired gauge-Higgs unification model
}

\author{
Yuta Orikasa ${ }^{a, *}$ \\ ${ }^{a}$ Institute of Experimental and Applied Physics, \\ Czech Technical University in Prague, \\ Husova 240/5, 11000 Prague 1, Czech Republic \\ E-mail: orikasa.kek@gmail.com
}

$\mathrm{SO}(5) \times \mathrm{U}(1) \times \mathrm{SU}(3)$ gauge-Higgs unification model inspired by $\mathrm{SO}(11)$ gauge-Higgs grand unification is constructed in the Randall-Sundrumwarped space. The 4D Higgs boson is identified with the Aharonov-Bohm phase in the fifth dimension. Fermion multiplets are introduced in the bulk in the spinor, vector and singlet representations of $\mathrm{SO}(5)$ such that they are implemented in the spinor and vector representations of $\mathrm{SO}(11)$. We discuss collider physics at International Linear Collider.

40th International Conference on High Energy physics - ICHEP2020

July 28 - August 6, 2020

Prague, Czech Republic (virtual meeting)

\footnotetext{
${ }^{*}$ Speaker
} 


\section{Introduction}

The Standard Model (SM) has been established at low energies. Almost all of the experimental results and observations have been consistent with the SM. However the SM is afflicted with the gauge hierarchy problem. The fundamental problem is the lack of a principle which regulates the Higgs sector.

One possible answer is the gauge-Higgs unification (GHU) in which the Higgs boson is identified with the Zero mode of the fifth demensional component of the gauge field. It appears as a fluction mode of the Aharonov-Bohm phase $\theta_{H}$ in the fifth dimentions.[1] The $S O(5) \times U(1)_{X} \times$ $S U(3)_{C}$ gauge theory in the Randall-Sundrum (RS) warped space has been proposed.[2] The model can be embedded in the $S O(11)$ gauge-Higgs grand unification, where the SM gauge group and quark-lepton content are incoporated into grand unified theory (GUT) in higher dimensional framework. In the model quark-lepton multiplets are introduced in the spinor, vector, and singlet representation of $S O(5)$. The model gives nearly the same phenomenology at low energiers as the SM.

\section{Gauge Higgs Unification model}

GHU predicts $Z^{\prime}$ bosons, which are the KK modes of $\gamma, Z$, and $Z_{R}$. They are mixed vector bosons of $U(1)_{X}, U(1)_{L} \subset S U(2)_{L}$, and $U(1)_{R} \subset S U(2)_{R}$ where $S U(2)_{L} \times S U(2)_{R} \subset S O(5)$. $Z^{\prime}$ bosons have broad widths and can be produced at $14 \mathrm{TeV}$ Large Hadron Collider (LHC). The current non-observation of $Z^{\prime}$ signals puts the limit $\theta_{H} \lesssim 0.11$. Distinct signals of the GHU can be found in $e^{-} e^{+}$collisions. Large parity violation appears in the couplings of quarks and leptons to KK gauge bosons, particularly to the $Z^{\prime}$ bosons. The interference effects between SM gauge bosons and $Z^{\prime}$ bosons can be clearly observed at $250 \mathrm{GeV}$ International Linear Collider (ILC).

\subsection{Forward-backward asymmetry}

The forward-backward asymmetry $A_{F B}^{f \bar{f}}\left(P_{e^{-}}, P_{e^{+}}\right)$is given by

$$
\begin{aligned}
A_{F B}^{f \bar{f}}\left(P_{e^{-}}, P_{e^{+}}\right) & =\frac{\sigma_{F}^{f \bar{f}}\left(P_{e^{-}}, P_{e^{+}}\right)-\sigma_{B}^{f \bar{f}}\left(P_{e^{-}}, P_{e^{+}}\right)}{\sigma_{F}^{f \bar{f}}\left(P_{e^{-}}, P_{e^{+}}\right)+\sigma_{B}^{f \bar{f}}\left(P_{e^{-}}, P_{e^{+}}\right)}, \\
\sigma_{F}^{f \bar{f}}\left(P_{e^{-}}, P_{e^{+}}\right) & =\int_{0}^{1} \frac{d \sigma^{f \bar{f}}}{d \cos \theta}\left(P_{e^{-}}, P_{e^{+}}, \cos \theta\right) d \cos \theta, \\
F \sigma_{B}^{f \bar{f}}\left(P_{e^{-}}, P_{e^{+}}\right) & =\int_{-1}^{0} \frac{d \sigma^{f \bar{f}}}{d \cos \theta}\left(P_{e^{-}}, P_{e^{+}}, \cos \theta\right) d \cos \theta,
\end{aligned}
$$

where $P_{e^{ \pm}}$denotes longitudinal polarization of $e^{ \pm} . P_{e^{ \pm}}=+1$ corresponds to purely right-handed $e^{ \pm}$. The amount of the deviation from the SM is characterized by

$$
\Delta_{A_{F B}}^{f \bar{f}} \equiv \frac{A_{F B, \mathrm{GHU}}^{f \bar{f}}}{A_{F B, \mathrm{SM}}^{f \bar{f}}}-1 .
$$




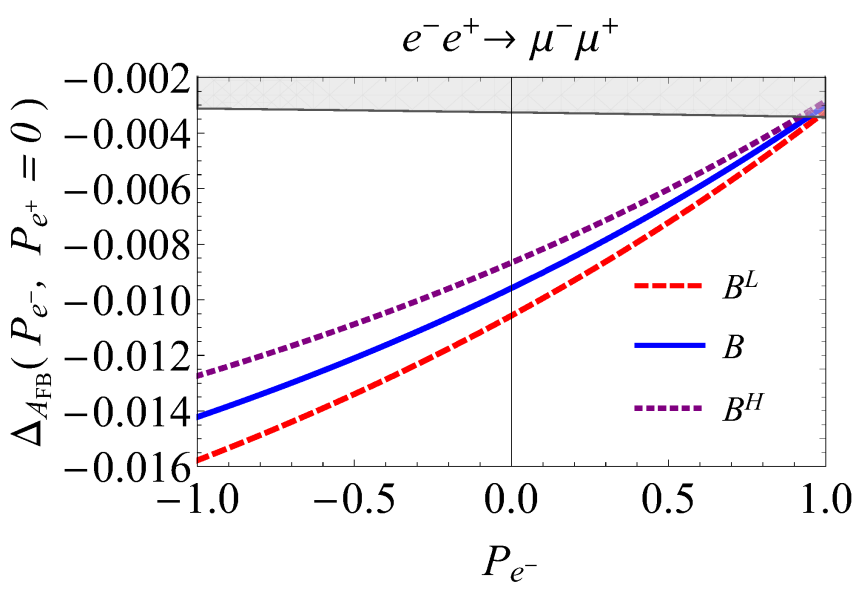

Figure 1: Forward-backward asymmetries $A_{F B}^{\mu^{-} \mu^{+}}$is shown. $\Delta_{A_{F B}}^{f \bar{f}}\left(P_{e^{-}}, P_{e^{+}}=0\right)$ in Eq. (2). The GHU $\left(\mathrm{B}^{\mathrm{L}}\right),(\mathrm{B}),\left(\mathrm{B}^{\mathrm{H}}\right)$ have different parameter sets. [3] The gray band represent the statistical error in the $\mathrm{SM}$ at $\sqrt{s}=250 \mathrm{GeV}$ with $250 \mathrm{fb}^{-1}$ data.

Forward-backward asymmetries $A_{F B}^{\mu^{-} \mu^{+}}$is shown in Figure 1. In forward-backward asymmetry for $f \bar{f}=\mu^{-} \mu^{+}$deviations from the SM are observed at ILC. The main reason for having these large effects lies in the fact that couplings of leptons and quarks to $Z^{\prime}$ bosons exhibit large parity violation. This is a special feature in GHU models formulated in the RS warped space. In GHU both left- and right-handed components of each lepton or quark are in one gauge multiplet, and each lepton or quark acquires a mass mainly through the Hosotani mechanism. It implies in the RS space that if the left-handed component is localized near the IR brane as in the GUT inspired GHU model, then the right-handed component is localized near the UV brane, and the left-handed component has a large coupling to $Z^{\prime}$ bosons as the overlap of wave functions becomes large.

The scenario of GHU leads to distinct signals in electron-positron collision experiments. Clear deviations from the SM should be observed in the early stage of ILC $250 \mathrm{GeV}$ experiments. In particular, GHU predicts strong dependence on the polarization of electron and positron beams, with which one can explore physics at the KK mass scale of $15 \mathrm{TeV}$.

\section{References}

[1] Y. Hosotani, Dynamical Mass Generation by Compact Extra Dimensions, Phys. Lett. B 126 (1983), 309-313

[2] S. Funatsu, H. Hatanaka, Y. Hosotani, Y. Orikasa and N. Yamatsu, GUT inspired $S O(5) \times$ $U(1) \times S U(3)$ gauge-Higgs unification, Phys. Rev. D 99 (2019) no.9, 095010

[3] S. Funatsu, H. Hatanaka, Y. Hosotani, Y. Orikasa and N. Yamatsu, Fermion pair production at $e^{-} e^{+}$linear collider experiments in GUT inspired gauge-Higgs unification, Phys. Rev. D 102 (2020) no.1, 015029 [arXiv:2006.02157 [hep-ph]]. 